\title{
iCompass at SemEval-2020 Task 12: From a Syntax-ignorant N-gram Embeddings Model to a Deep Bidirectional Language Model
}

\author{
Abir Messaoudi \\ iCompass / Tunisia \\ abir@icompass.com
}

\author{
Hatem Haddad \\ iCompass / Tunisia \\ hatemeicompass.tn
}

\author{
Moez Ben Hajhmida \\ iCompass / Tunisia \\ moezdicompass.tn
}

\begin{abstract}
We describe our submitted system to the SemEval 2020. We tackled Task 12 entitled "Multilingual Offensive Language Identification in Social Media", specifically subtask 4A-Arabic. We propose three Arabic offensive language identification models: Tw-StAR, BERT and BERT+BiLSTM. Two Arabic abusive/hate datasets were added to the training dataset: L-HSAB and T-HSAB. The final submission was chosen based on the best performances which was achieved by the BERT+BiLSTM model.
\end{abstract}

\section{Introduction}

With the freedom of expression privilege granted after the Arab countries revolution, sensitive topics such as religion and politics have become popular and widely discussed across social media platforms. However, on the down side, offensive language spreads easily. Indeed, recent events; like Persian's gulf crisis, the parliamentary-presidential elections held in Tunisia or a football game between two Arabic clubs; caused intensive debates, most of them took place on social media networks leading to a high emergence of offensive speech. This evokes the need for tools to identify such online offensive language content.

Analyzing Arabic offensive language is significantly challenging due to the complex nature and morphology of the Arabic language. Furthermore, Arabic language in Social Media is mostly informal and written in Arabic dialects.

We describe our participation in SemEval 2020 Task 12 entitled "Multilingual Offensive Language Identification in Social Media" Zampieri et al. (2020), specifically Task 12 4A-Arabic: "Arabic Offensive Language Identification in Social Media" Mubarak et al. (2020) under the team name "iCompass". The task requires to distinguish between offensive (containing any form of non-acceptable language (profanity)) and non-offensive posts.

The remainder of the paper is organized as follows: in Section 2, we introduce L-HSAB and T-HSAB datasets. In Section 3, we describe the preprocessing step. Section 4 introduces the learning strategies and datasets used in the presented models. Results are reviewed and discussed in Section 5 while Section 6 concludes the study.

\section{Arabic offensive language datasets}

The proposed dataset for the SemEval task 12 subtask A-Arabic is composed mainly by Egyptian dialect comments and few other dialects like Libyan, Sudanese, Syrian, etc. Some comments, after the preprocessing phase, in different dialects are given in Table $1^{1}$.

Consequently, in addition to the dataset proposed by SemEval Task 12 4A-Arabic, we used two other Arabic offensive language datasets : L-HSAB and T-HSAB.

L-HSAB (a Twitter dataset about abusive speech (AS) and hate speech (HS)) is introduced in Mulki et al. (2019a) as a benchmark dataset for automatic detection of online Levantine offensive language. The

\footnotetext{
This work is licensed under a Creative Commons Attribution 4.0 International Licence. Licence details: http:// creativecommons.org/licenses/by/4.0/.

${ }^{1}$ Comments numbers $700,1088,5$ and 2439 of the training set.
} 


\begin{tabular}{|c|c|c|}
\hline Dialect & Comment & English translation \\
\hline Egyptian & مش خلاص كدة. & ev my love isn't en \\
\hline North African & ..... & $\begin{array}{l}\text { Stay like that keep nagging till tomor- } \\
\text { row.. }\end{array}$ \\
\hline Leva & يا بكون بحياتك الأهم يا إما ما بدي أكون & $\begin{array}{l}\text { I shall be at the highest priority in your } \\
\text { life, or I don't wanna be in your life. }\end{array}$ \\
\hline Golf & يا حســــــايف قو لتي لك يـا حبيبي يو م قلبي & $\begin{array}{l}\text { Oh damage, I called you my beloved } \\
\text { before I know the real you. }\end{array}$ \\
\hline
\end{tabular}

Table 1: Examples of comments in different dialects.

dataset is composed of $5.8 \mathrm{~K}$ tweets, manually annotated as Normal, Abusive and Hate. The high obtained values of agreement without chance correction and inter-annotator agreement indicated the reliability of the dataset. The inter-rater agreement metric denoted by Krippendorff's alpha $(\alpha)$ was $76.5 \%$ and indicated the consistency of the annotations.

T-HSAB was constructed out of Tunisian comments harvested from different social media platforms and introduced in Haddad et al. (2019) as a benchmark dataset for automatic detection of online Tunisian offensive language. The dataset is composed of $6 \mathrm{~K}$ tweets, manually annotated as Normal, Abusive and Hate. It has high values of agreement without chance correction and inter-annotator agreement and Krippendorff's alpha $(\alpha)$ value of $75 \%$.

\section{Data Preprocessing}

Data was preprocessed by cleaning the tweets from the social media-inherited symbols such as $(\mathrm{Rt},<\mathrm{LF}>$ and @), URLs, Usernames, dates, retweets, symbols, punctuations, emojis and non-Arabic characters, in order to remove noise and to get the Arabic text only.

Table 2 shows an example of the comment number 2372 of the training set, before and after preprocessing.

\begin{tabular}{|c|c|}
\hline $\begin{array}{l}\text { Before } \\
\text { After }\end{array}$ & 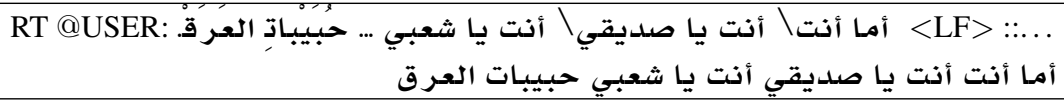 \\
\hline
\end{tabular}

Table 2: Example of a comment before and after preprocessing phase.

\section{Used models and Learning Strategies}

In this section, we describe the learning strategies and the different architectures used. The mechanism of each strategy is briefly reviewed. To accomplish this mission, we have used three classification approaches.

\subsection{Tw-StAR}

Tw-StAR is a syntax-ignorant n-gram embeddings model used in sentiment analysis of several Arabic dialects Mulki et al. (2019b). Tw-StAR's embeddings are composed and learned using an unordered composition function and a shallow neural model. The model performed state of art results at Semeval2017 Task 4: "Sentiment Analysis in Twitter" Mulki et al. (2017) including Arabic language and SemEval-2018 Task 1: "Affect in Tweets", subtask Ec "Detecting Emotions (multi-label classification)" Mulki et al. (2018).

In addition, we have used an offensive lexicon extracted from L-HSAB and T-HSAB datasets. The خائن، جز مـة، عاهرة، ساقط، that mean Thief, traitor... We have combined the predictions of our classifier with the results of the lexicon-based method to have a hybrid solution. This mixed solution have decreased the results since it can neither identify negations nor detect non-offensive comments that contain offensive words, example in table 3. 


\section{Arabic $\quad$... ده كان انسان طيب و متقي مش حر امي وخاين زي ماكانو يقو لو \\ English $\quad . .$. this was a good and fearful person, not a thief and a traitor as they said}

Table 3: Example of a non offensive comment that contains offensive words.

Tw-StAR+Lexicon approach has classified this comment as offensive because it contains حر امين خاين, which means Thief and Traitor, that appear in the lexicon of offensive words and confuse the prediction results of this type of sentences. This approach decreased the performance results as given in Table 5.

\subsection{BERT}

Context-free models such as word2vec or GloVe generate a single word embedding representation for each word in the vocabulary. Differently, contextual models generate a representation of each word based on the other words in the sentence. For this reason, we selected the Bidirectional Encoder Representations from Transformers (BERT) Devlin et al. (2019) as a contextual language model in its light multilingual version as an embedding technique.

BERT is a deep bidirectional language model, pretrained on large corpora (BooksCorpus: $800 \mathrm{M}$ words, and Wikipedia: 2,500M words), that can be fine-tuned to solve many NLP tasks such as named entity recognition (NER), question answering (QA) and text classification Devlin et al. (2019).

We used the BERT base multilingual cased model, with 12 transformer layers, 12 attention heads and $110 \mathrm{M}$ parameters. We used the already pretrained model and trained the classifier to predict the probabilities of the labels (OFF or NOT) tuning different values of hyper parameters in order to have the best performances. Values for fine-tuning are stated in Table 4.

\subsection{BERT + BiLSTM}

The dataset was tokenized using the BERT tokenizer mapping Arabic words to their indexes. BERT embedding matrix was used at the embedding layer level. Then, BILSTM model was used as classifier in order to predict label's probabilities.

\begin{tabular}{|l|lll|}
\hline & Tw-StAR & BERT & BERT+BILSTM \\
\hline Batch size & 128 & 8 & 16 \\
Sequence length & - & 128 & 128 \\
Epochs & 6 & 3 & 6 \\
N-grams (N) & 8 & - & - \\
\hline
\end{tabular}

Table 4: Hyper parameters used during training phase.

\section{Results and Discussion}

Three datasets are provided by SemEval 2020 Task 12 subtask 4A-Arabic: TRAIN (7000 comments) for training models, DEV (1000 comments) for tuning models, and TEST (2000 comments) for the official evaluation.

Data was preprocessed using regular expressions recognition and regular expressions substitution provided by the re Python module ${ }^{2}$. Having the data preprocessed and the features extracted, training dataset was splitted into $80 \%$ for training and $10 \%$ for cross-validation. We have trained our Tw-StAR, BERT, and BiLSTM models on the DEV set.

Table 5 lists the results of the four classification architectures: Tw-StAR, Tw-StAR+Lexicon, BERT, and BERT+BiLSTM. As a result, a slight improvement was achieved by BERT when compared to the Tw-StAR baseline and BiLSTM by achieving the best performances with a macro F-score (Macro F1) of 0.825 and an accuracy of 0.898 .

\footnotetext{
${ }^{2}$ https://docs.python.org/3/library/re.html
} 


\begin{tabular}{|l|lll|}
\hline Model & Accuracy & Micro F1 & Macro F1 \\
\hline Tw-StAR & 0.813 & 0.813 & 0.331 \\
Tw-StAR + Lexicon & 0.818 & 0.818 & 0.332 \\
BERT & 0.821 & 0.821 & 0.791 \\
BERT+BiLSTM & $\mathbf{0 . 8 9 8}$ & $\mathbf{0 . 8 9 8}$ & $\mathbf{0 . 8 2 5}$ \\
\hline
\end{tabular}

Table 5: Results on the SemEval Devset.

In order to improve the performances, we used T-HSAB and L-HSAB datasets for training in addition to the SemEval 2020 Task 12 subtask 4A-Arabic provided training dataset. Characteristics of each dataset are given in Table 6.

\begin{tabular}{|l|llll|}
\hline Dataset & Dialect & NOT & OFF & Total \\
\hline L-HSAB & Levantine & 3650 & 2196 & 5846 \\
T-HSAB & Tunisian & 3835 & 2204 & 6039 \\
SemEval 2020 & mostly Egyptian & 5666 & 1334 & 7000 \\
Total & - & 13151 & 5734 & 18885 \\
\hline
\end{tabular}

Table 6: Datasets used during the training phase.

Considering the results stated in Table 5 and Table 7, the supervised learning-based model with BiLSTM algorithm achieved the best average F-score (micro and marco) performances compared to the BERT base and the Tw-StAR model. Hence, BERT+BiLSTM was used to provide the TEST set classification results for the final submission.

\begin{tabular}{|l|lll|}
\hline Training dataset & Accuracy & Micro F1 & Macro F1 \\
\hline SemEval & 0.885 & 0.885 & 0.784 \\
SemEval+L-HSAB & $\mathbf{0 . 9 0 0}$ & $\mathbf{0 . 9 0 0}$ & 0.794 \\
SemEval+L-HSAB + T-HSAB & 0.898 & 0.898 & $\mathbf{0 . 8 2 5}$ \\
\hline
\end{tabular}

Table 7: Results on SemEval Devset according to the training dataset.

Table 8 reviews the official results of iCompass system against the top three ranked systems.

\section{Conclusion and Future work}

Three classification architectures were used to identify offensive language Arabic tweets. The best F-score macro results were obtained by the BiLSTM classification architecture using BERT base multilingual word embedding which was selected for the final submission.

\section{References}

Jacob Devlin, Ming-Wei Chang, Kenton Lee, and Kristina Toutanova. 2019. BERT: Pre-training of deep bidirectional transformers for language understanding. In Proceedings of the 2019 Conference of the North American Chapter of the Association for Computational Linguistics: Human Language Technologies, Volume 1 (Long and Short Papers), pages 4171-4186, Minneapolis, Minnesota, June.

Hatem Haddad, Hala Mulki, and Asma Oueslati. 2019. T-HSAB: A tunisian hate speech and abusive dataset. In Kamel Smaili, editor, Arabic Language Processing: From Theory to Practice - 7th International Conference, ICALP 2019, Nancy, France, October 16-17, 2019, Proceedings, volume 1108 of Communications in Computer and Information Science, pages 251-263. Springer.

Hamdy Mubarak, Ammar Rashed, Kareem Darwish, Younes Samih, and Ahmed Abdelali. 2020. Arabic offensive language on twitter: Analysis and experiments. arXiv preprint arXiv:2004.02192. 


\begin{tabular}{|l|ll|}
\hline Team & Rank & macro F1-score \\
\hline ALAMI Hamza & 1 & 0,9017 \\
alt & 2 & 0,9015 \\
Galileo & 3 & 0,8988 \\
iCompass & 14 & 0,8519 \\
\hline
\end{tabular}

Table 8: Ranking and results on SemEval Test set.

Hala Mulki, Hatem Haddad, Mourad Gridach, and Ismail Babaoğlu. 2017. Tw-star at semeval-2017 task 4: Sentiment classification of arabic tweets. In Proceedings of the 11th International Workshop on Semantic Evaluation, pages 664-669, Vancouver, Canada, August.

Hala Mulki, Chedi Bechikh Ali, Hatem Haddad, and Ismail Babaoğlu. 2018. Tw-StAR at SemEval-2018 task 1: Preprocessing impact on multi-label emotion classification. In Proceedings of The 12th International Workshop on Semantic Evaluation, pages 167-171, New Orleans, Louisiana, June.

Hala Mulki, Hatem Haddad, Chedi Bechikh Ali, and Halima Alshabani. 2019a. L-HSAB: A Levantine twitter dataset for hate speech and abusive language. In Proceedings of the Third Workshop on Abusive Language Online, pages 111-118, Florence, Italy, August. Association for Computational Linguistics.

Hala Mulki, Hatem Haddad, Mourad Gridach, and Ismail Babaoğlu. 2019b. Syntax-ignorant n-gram embeddings for sentiment analysis of Arabic dialects. In Proceedings of the Fourth Arabic Natural Language Processing Workshop, pages 30-39, Florence, Italy, August.

Marcos Zampieri, Preslav Nakov, Sara Rosenthal, Pepa Atanasova, Georgi Karadzhov, Hamdy Mubarak, Leon Derczynski, Zeses Pitenis, and Çağrı Çöltekin. 2020. SemEval-2020 Task 12: Multilingual Offensive Language Identification in Social Media (OffensEval 2020). In Proceedings of SemEval. 